\title{
Perfil toxicológico e oxidativo de comerciantes de Tacacá expostos ao cianeto no município de Belém/PA
}

\author{
Toxicological and oxidative profile of Tacacá merchants exposed to cyanide in the \\ municipality of Belém/PA
}

\section{Perfil toxicológico y oxidativo de los comerciantes de Tacacá expuestos al cianuro en el municipio de Belém/PA}

Amanda Ribeiro Leal ${ }^{1 *}$, Brenda Caroline dos Santos Pinheiro ${ }^{1}$, Ingryd Rayssa Duarte Lobato', Cláudia Simone Baltazar de Oliveira².

\section{RESUMO}

Objetivo: Avaliar a exposição ao cianeto pela via inalatória de comerciantes de Tacacá no município de Belém/Pará. Métodos: O estudo foi observacional do tipo transversal e analítico realizado em 2019 em amostra biológica (sangue) de comerciantes de Tacacá e de grupo controle. Foi realizado a dosagem de cianeto livre ( $\mathrm{HCN})$ e de Metahemoglobina $(\mathrm{MeHb})$ por meio da espectrofotometria Uv (Ultravioleta). Os dados foram avaliados através de estatística descritiva além de testes de análise de variância e correlação de Pearson, adotando $p \leq 0,05$. O estudo foi aprovado pelo Comitê de Ética de Pesquisa. Resultados: $A$ concentração média de HCN livre das amostras da população exposta foi de 1,32 mg/L e não-exposta de $1,29 \mathrm{mg} / \mathrm{L}$ classificada como exposição moderada $(\mathrm{p}=0,0068)$. Os valores da dosagem de $\mathrm{MeHb}$ foram de $2,7 \%$ para o grupo exposto e 2,6\% para grupo não-exposto encontrando-se dentro dos parâmetros. Conclusão: A correlação entre $\mathrm{MeHb}$ e $\mathrm{HCN}$ no grupo exposto, apesar de não mostrar significância estatística, causa preocupação pela forma que ambos se relacionam. Foi possível observar que quanto maior o nível de exposição ao cianeto, maior serão os níveis de MeHb.

Palavras-Chave: Mandioca, Ácido cianídrico, Cianeto.

\begin{abstract}
Objective: The evaluate exposure to cyanide inhalation via from to Tacacá traders in the municipality of Belém, Pará state. Methods: The cross-sectional and analytical observational study was conducted in 2019 on a biological sample (blood) from Tacacá traders and control group. The cyanide free (HCN) and Metahemoglobin ( $\mathrm{MeHb}$ ) were measured by spectrophotometry Uv (Ultraviolet). Data stood evaluated using descriptive statistics and tests of analysis of variance and Pearson correlation, adopting $p \leq 0.05$. The study was approved the Research Ethics Committee. Results: Mean HCN free concentration of exposed population samples remained $1.32 \mathrm{mg} / \mathrm{L}$ and unexposed $1.29 \mathrm{mg} / \mathrm{L}$ is classified as moderate exposure, $\mathrm{p}$ value $=$ 0.0068 . MeHb dosage values was $2.7 \%$ for the exposed group and $2.6 \%$ for the unexposed group, meeting the parameters established. Conclusion: The correlation among $\mathrm{MeHb}$ and $\mathrm{HCN}$ in the exposed group, although not statistical significance. It was observed that the higher cyanide exposure level and higher MeHb levels.
\end{abstract}

Keywords: Cassava, Hydrocyanicacid, Cyanide.

\section{RESUMEN}

Objetivo: Evaluar la exposición al cianuro por medio de la vía inhalatoria de comerciantes de Tacacá en el municipio de Belém/Pará. Métodos: El estúdio fue mediante a la observación del tipo transversal y analítica realizada con amuestra biológica (la sangre) de comerciantes del producto Tacacá y del grupo de control, en el año 2019. Para tanto, a través de la espectrofotometría Uv (Ultavioleta) fue realizada la medición del cianuro libre $(\mathrm{HCN})$ y también de la Metahemoglobina $(\mathrm{MeHb})$, para colectar datos. De este modo, tras la medición,

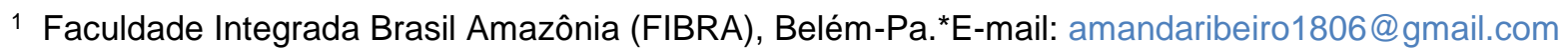

2 Universidade Federal do Pará (UFPA), Belém-PA. 
los datos fueron evaluados por medio de la estadística descriptiva, además de testes de análisis de variancia y correlación de Pearson, adoptando $p \leq 0,05$. Tras los procedimientos supramencionados, el estudio obtuvo aprobación por el Comité de Ética de investigación. Resultados: La concentración media de HCN libre de las amuestras de la población expuesta fue de 1, $32 \mathrm{mg} / \mathrm{l}$ se clasifica como exposición moderada $(p=0,0068)$. Los valores de la medición de MeHb fueron de 2,7\% para el grupo expuesto y de 2,6\% para el grupo no expuesto, o sea, encontrándose dentro de los parámetros estabelecidos. Conclusión: En este sentido, la correlación entre MeHb y HCNen el grupo no expuesto, aunque no tenga se mostrado significante estadísticamente, causa preocupación por la forma como ambos se relacionan. En suma, fue posible observar que, cuanto mayor el nivel de exposición al cianuro, mayor serán los niveles de MeHb.

Palabras clave: Yuca, Ácido cianhídrico, Cianuro.

\section{INTRODUÇÃO}

No Estado do Pará, a mandioca (Manihot esculenta Crantz) destaca-se entre os alimentos presente na alimentação de sua população e, é ingerida principalmente na forma de farinha, sendo também utilizada como elemento fundamental para a elaboração de diversos pratos típicos, dentre eles, o tacacá, preparação constituída por subprodutos da mandioca (tucupi e/ou goma), camarão seco, jambu e outros temperos; sua comercialização é muito comum gerando renda e emprego no município (CONCEIÇÃO EC, 2016).

A preocupação com relação à esta iguaria e seus componentes, está sendo difundida em vários estudos por se tratar de um agente altamente cianogênico (BORGES MF, et al., 2002; DIAS VL, et al., 2016). Segundo o Ministério da Saúde, em 2014, foi registrado no Sistema de Informação de Agravos de Notificação - SINAN um total de 89.587 atendimentos por intoxicação. Do total dessas intoxicações, apenas 719 casos estão relacionados à exposição a plantas tóxicas.

A exposição ao cianeto pode ser considerada uma intoxicação rara, porém de extrema gravidade, realçando que tal intoxicação se manifesta de forma mais frequente do que o esperado devido a falta de registro e subnotificação.

Os casos de intoxicação por essa substância podem ocorrer pela via oral e inalatória. O cianeto é extremamente relevante na área toxicológica, devido aos seus mecanismos toxicocinéticos e toxicodinâmicos, capazes de causar danos irreversíveis aos organismos expostos e até mesmo a morte (SOUSA AB, 2004; BRASIL, 2015).

O cianeto, produto decorrente da reação química entre a linamarina e linamarase (enzima que degrada o composto linamarina), é considerado para os organismos biológicos uma substância química de elevada toxicidade, capaz de causar danos mitocondriais e déficits na respiração celular.

Além disso, nos eritrócitos humanos, o cianeto é substância considerada metahemoglobinizante interferindo na reação da oxidação e redução do ferro, o que impede a ligação do átomo de O2 (oxigênio) a molécula de hemoglobina na hemácia, produzindo assim hipóxia nos tecidos. Além disso, também são capazes de dificultar a atividade da anidrase carbônica, o que confere ao indivíduo exposto maior susceptibilidade a acidose metabólica (ARAUJO JSP e LOPES CA, 2009; SHIBAMOTO T e BJELDANE LF, 2014; AZEVEDO FA, et al., 2015).

Estudos já publicados citam diversas doenças associadas à ingestão frequente de alimentos contento um alto teor de ácido cianídrico ( $\mathrm{HCN})$, que é um composto derivado do Cianeto (CN-). Dentre as principais doenças podemos destacar a doença de konzo, bócio endêmico e câncer no estômago (TEÓFILO TJS et al., 2004; REZENDE ALS, et al., 2006; LOPES AM, 2011).

No entanto, em decorrência da manipulação do tucupi, os comerciantes podem estar sendo expostos ao cianeto através dos vapores emitidos, sem ter o conhecimento dos possíveis riscos trazidos à saúde acidentalmente.

Devido ausência de estudos nessa temática, nossa proposta é investigar a exposição dos comerciantes de Tacacá no município de Belém ao cianeto. Esse estudo visa analisar a concentração de cianeto nas amostras biológicas, levando em consideração os riscos de danos biológicos decorrentes da exposição, e assim prevenir prováveis patologias inerentes ao químico. 


\section{MÉTODOS}

A metodologia escolhida para o referido trabalho foi de estudo observacional do tipo transversal analítico, que foi realizado durante o ano de 2019 em amostra biológica de comerciantes de Tacacá e de grupo controle do município de Belém-PA.

O estudo foi realizado com comerciantes de tacacá localizados nos distritos que se encontram no município de Belém-Pará. Segundo o Anuário Estatístico do Município de Belém (2012) os 71 bairros de Belém são subdivididos em oito distritos, sendo eles: DAMOS (Distrito Administrativo de Mosqueiro), DAOUT (Distrito Administrativo de Outeiro), DAICO (Distrito Administrativo de Icoaraci), DABEN (Distrito Administrativo do Benguí), DAENT (Distrito Administrativo do Entroncamento), DASAC (Distrito Administrativo do Sacramenta), DABEL (Distrito Administrativo de Belém), DAGUA (Distrito Administrativo do Guamá).

A amostra para o estudo foi do tipo probabilística, aleatória simples. Por ser uma atividade informal onde não há dados e/ou estudos similares que possam quantificar o número de profissionais no município, foi utilizado como critério de seleção a divisão político administrativa do município de Belém, onde foram sorteados $10 \%$ do total de bairros da cidade, ou seja, 8 bairros. A partir disto, os comerciantes de tacacá foram incluídos na pesquisa por demanda espontânea, sendo necessário o máximo de 2 voluntários por bairro, sendo 1 comerciante e 1 indivíduo não exposto, totalizando 16 participantes.

Apresentamos como critério de inclusão da pesquisa homens e mulheres que apresentaram como principal atividade laboral a comercialização do Tacacá, há no mínimo um ano e maiores de 18 anos. Foram excluídos do estudo aqueles comerciantes que no dia da visita declararam não ter como principal atividade laboral a comercialização do tacacá, fumantes e produtores de farinha, já que essas características podem levar a vieses dos resultados no presente estudo.

Utilizamos para a pesquisa amostra de sangue, a qual foi coletada por técnicos experientes tanto no laboratório de análises clínicas da Faculdade Integrada Brasil Amazônia, quanto na casa dos participantes que não puderam comparecer na instituição. Após a coleta, o sangue foi colocado em tubo com EDTA (ácido etilenodiaminotetra-acético) e mantido em refrigeração até o momento da análise. Os testes foram realizados nos Laboratórios da Instituição. O material biológico foi devidamente descartado segundo a RDC nํ 306 de 7 de dezembro de 2004.

As amostras de sangue foram submetidas a técnicas para a dosagem de metahemoglobina e Cianeto livre. Na primeira técnica citada seguiu o protocolo de Naoum PC et al. (2004) através da utilização de saponina $1 \%$, solução de tampão fosfato M60 e M15. A segunda seguiu a metodologia descrita por Cooke RD (1978) que posteriormente foi adaptada por Essers AJA et al. (1993) utilizando solução alcoólica de ácido fosfórico $0,1 \mathrm{~mol}$, somado ao tampão Ph6, cloramina T e o reagente de cor. Por fim ambas foram submetidas à espectrofotometria Uv.

A análise dos dados é do tipo quantitativo, apresentado em gráficos e tabelas. Os elementos do conjunto de dados foram avaliados através de estatística descritiva como média e desvio padrão, além de testes de análise de variância como, Teste t student e correlação de Pearson. O programa estatístico de escolha foi o Bioestat 5.3, adotando $p \leq 0,05$.

Durante as visitas aos comerciantes e ao grupo não exposto, foi apresentado o projeto de maneira clara e detalhada. Os indivíduos foram convidados a participar da pesquisa após aceitação do Termo de Consentimento Livre e Esclarecido (TCLE - apêndice A), devidamente assinado, em seguida foi aplicado um questionário por entrevista (apêndice B) para coleta de informações com intuito de traçar o perfil sócio demográfico dos participantes. O protocolo deste estudo foi elaborado de acordo com as normas da resolução 466/2012 e aprovado pelo CEP da Faculdade Integrada Brasil Amazônia (FIBRA) de acordo com o parecer ético no 3.632.586 de 09 de outubro de 2019 (anexo I) sendo este um projeto proveniente à iniciação científica da Instituição.

\section{RESULTADOS}

Dos dezesseis participantes selecionados para o presente estudo, 50\% das amostras analisadas correspondem à população exposta e outros $50 \%$ a população não exposta, de ambos os sexos, demonstrado 
na tabela abaixo a descrição das características sócio demográficos da população de estudo e controle (Tabela 1).

De acordo com a Tabela 1, A idade variou entre 19 e 82 anos. A maior representatividade entre as faixas etárias foi a de 25 a 64 anos, representando $62,5 \%$ para ambos os grupos. Na variável escolaridade, tivemos 3 grupos: os que declararam ter estudado mais de 11 anos (62,5\% população exposta e 50\% população não exposta); seguido o grupo de 8 anos estudados (ambos 25\%) e os que tiveram mais que 14 anos estudados (12,5\% e $25 \%$ exposto e não exposto respectivamente).

Cem por cento (100\%) do grupo exposto declarou ter como principal atividade laboral a comercialização do tacacá. Já o grupo não exposto se dividiu nas seguintes categorias: estudante (6,25\%), do lar (6,25\%) e outros $(37,5)$. Quanto ao estado civil, $75 \%$ do grupo exposto e $62,5 \%$ do grupo não exposto alegou estar solteiro, enquanto $25 \%$ grupo exposto e $37,5 \%$ grupo não exposto estar casado, descrito na Tabela 1.

Tabela 1 - Características sócio demográficas da população de estudo envolvida com a comercialização do tacacá e da população não exposta, 2019.

\begin{tabular}{|c|c|c|c|c|}
\hline \multirow{2}{*}{$\begin{array}{c}\text { Perfil } \\
\text { Sócio demográfico } \\
\text { Sexo }\end{array}$} & \multicolumn{2}{|c|}{ Frequência } & \multicolumn{2}{|c|}{ Percentual (\%) } \\
\hline & Exposto & n/ exposto & Exposto & n/ exposto \\
\hline Homem & 4 & 4 & 50 & 50 \\
\hline Mulher & 4 & 4 & 50 & 50 \\
\hline \multicolumn{5}{|l|}{ Faixa etária (anos) } \\
\hline $\begin{array}{c}19 \text { a } 24 \\
\text { (Jovem adulto) }\end{array}$ & 1 & 1 & 12,5 & 12,5 \\
\hline $\begin{array}{l}25 \text { a } 64 \\
\text { (Adulto) }\end{array}$ & 5 & 5 & 62,5 & 62,5 \\
\hline $\begin{array}{l}\text { A partir de } 65 \\
\text { (Idoso) }\end{array}$ & 2 & 2 & 25 & 25 \\
\hline \multicolumn{5}{|l|}{$\begin{array}{c}\text { Grau de Instrução (anos de } \\
\text { estudo) }\end{array}$} \\
\hline Até 8 anos estudados & 2 & 2 & 25 & 25 \\
\hline Até 11 anos estudados & 5 & 4 & 62,5 & 50 \\
\hline >14 anos estudos & 1 & 2 & 12,5 & 25 \\
\hline \multicolumn{5}{|l|}{ Ocupação } \\
\hline Comerciantes de tacacá & 8 & 0 & 100 & - \\
\hline Do lar & - & 1 & - & 6,25 \\
\hline Estudante & - & 1 & - & 6,25 \\
\hline $\begin{array}{c}\text { Outros (Motorista, } \\
\text { Psicopedagoga, Ass. } \\
\text { Administrativo, Aposentado, } \\
\text { Coletador de resíduos) }\end{array}$ & - & 6 & - & 37,5 \\
\hline \multicolumn{5}{|l|}{ Estado civil } \\
\hline Solteiro & 6 & 5 & 75 & 62,5 \\
\hline Casado & 2 & 3 & 25 & 37,5 \\
\hline
\end{tabular}

Legenda: *Comparação entre os itens das variáveis sócio- demográficas. Fonte: Leal AR, et al., 2019.

Quando questionados sobre o tempo de atividade laboral, $87,5 \%$ da população exposta declararam ter mais de 37 meses trabalhados, enquanto 12,5\% afirmaram ter trabalhado menos que 36 meses. Ao que diz respeito sobre a jornada de trabalho $50 \%$ relatou que trabalha até 6 hrs diárias, entretanto, outros $50 \%$ afirmaram trabalhar mais que 7hrs por dia. Tais dados estão representados na Figura 1. 
Figura 1 - Dados laborais do grupo exposto envolvidos com a comercialização do tacacá em Belém-Pa, 2019.

Tempo de atividade laboral

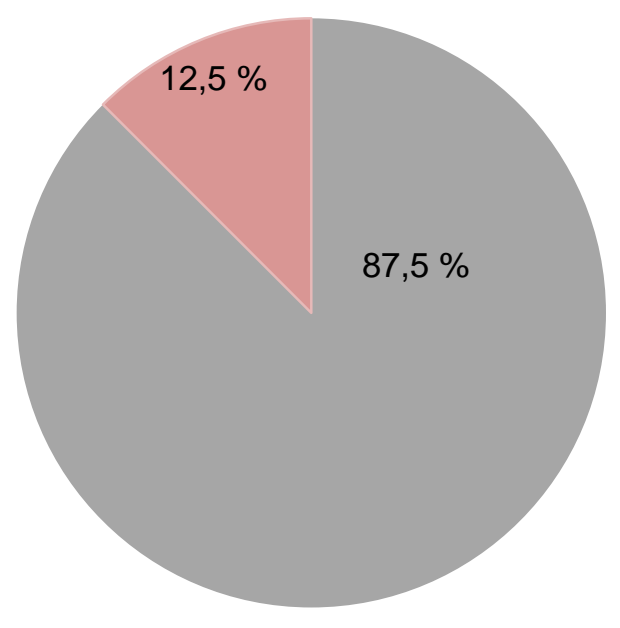

$\llbracket 37$ meses $\quad \approx 36$ meses

\section{Jornada de trabalho}

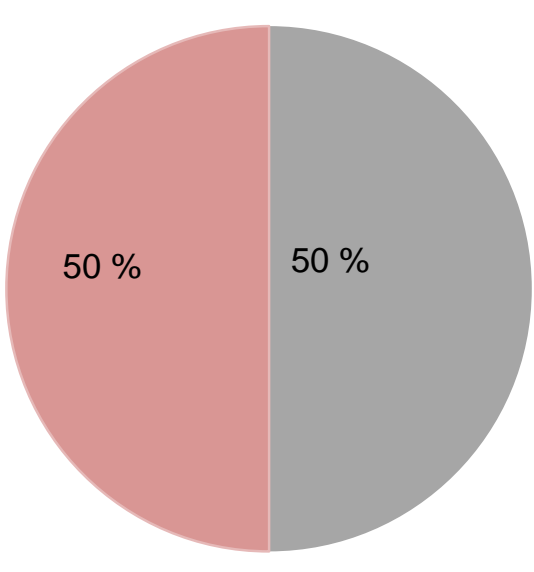

$\square$ Até 6 horas $\square>7$ horas

Legenda: *Relação entre o tempo de atividade laboral e jornada de trabalho do grupo exposto.

Fonte: Leal AR, et al., 2019.

Na Tabela 2 está representado o perfil hematológico, bioquímico e oxidativo dos participantes inclusos na pesquisa no município de Belém/Pá no ano de 2019. Foram avaliadas as concentrações de hemoglobina $(\mathrm{Hb})$ e hematócrito $(\mathrm{HT})$ dentre os biomarcadores hematológicos.

A média da $\mathrm{Hb}$ dos participantes expostos foi de $13,3 \mathrm{~g} / \mathrm{dL}$, em contrapartida o resultado da população não exposta foi de $13,8 \mathrm{~g} / \mathrm{dL}$. No HT, foi observado valor da média de $40,1 \%$ e de $41,9 \%$ da população exposta e população não exposta, respectivamente.

$\mathrm{Na}$ avaliação da $\mathrm{Hb}$ apenas o grupo exposto apresentou $25 \%$ dos valores abaixo dos intervalos de referência para $\mathrm{Hb}$. No parâmetro da concentração de $\mathrm{HT}$, ambos os grupos apresentaram alterações, sendo que $25 \%$ da população exposta e $12,5 \%$ da população não exposta apresentaram níveis abaixo da referência, sendo esses últimos dados não demonstrados em tabela.

A média do colesterol total (CT) obtida dos resultados dos participantes foi de 158,2 $\mathrm{mg} / \mathrm{dL}$ para grupo exposto e de $155,5 \mathrm{mg} / \mathrm{dL}$ para o grupo não exposto. Os valores da média dos triglicerídeos (Tri) obtidos para o grupo exposto e não exposto foram de $227,5 \mathrm{mg} / \mathrm{dL}$ e $145,6 \mathrm{mg} / \mathrm{dL}$, respectivamente. Nos últimos critérios demonstrados, o CT não evidenciou alterações nos grupos, enquanto o Tri encontrava-se acima dos limites de normalidade.

Na avaliação do TGP (Transaminase glutâmica pirúvica) e do TGO (Transaminase glutâmica oxalacética) foi obtida a média de $23 \mathrm{U} / \mathrm{L}$ para grupo exposto e 43,7 U/L para grupo não exposto; e 27,3 U/L para grupo exposto e 36,8 $\mathrm{U} / \mathrm{L}$ para grupo controle $\mathrm{U} / \mathrm{L}$, respectivamente.

O nível médio de uréia para grupo exposto foi de $29,1 \mathrm{mg} / \mathrm{dL}$ e $31,3 \mathrm{mg} / \mathrm{dL}$ para grupo não exposto; creatina de $0,8 \mathrm{mg} / \mathrm{dL}$ para grupo exposto e $0,7 \mathrm{mg} / \mathrm{dL}$ para o grupo controle, sendo assim, a média desses marcadores não apresentou alterações.

$\mathrm{Na}$ avaliação das proteínas totais a média foi de $7,1 \mathrm{~g} / \mathrm{dL}$ para grupo exposto e de $6,6 \mathrm{~g} / \mathrm{dL}$ para grupo não exposto, ambos os grupos não se encontravam acima da normalidade. Por fim na avaliação oxidativa, a dosagem da Metahemoglobina (Mhb) se encontrou dentro dos parâmetros estabelecidos (abaixo que 4\%), de $2,7 \%$ para grupo exposto e 2,6\% para grupo não exposto (valores de referência estabelecidos por Naoum PC et al., 2004). 
Tabela 2 - Perfil hematológico, bioquímico e oxidativo de comerciantes de tacacá expostos ao HCN e grupo não expostos localizados nos distritos de Belém-Pará, 2019.

\begin{tabular}{|c|c|c|c|}
\hline Biomarcador & $\dot{\mathrm{X}} \pm \mathrm{DP}$ & $\dot{X} \pm D P$ & Valor de Referência \\
\hline Hematológico & Expostos & Controle & \\
\hline Hemoglobina $\mathrm{g} / \mathrm{dL}$ & $13,3 \pm 2,0$ & $13,8 \pm 1,7$ & $\begin{array}{l}H: 13-18 \\
M: 11-16\end{array}$ \\
\hline Hematócrito \% & $40,1 \pm 4,8$ & $41,9 \pm 5,4$ & $\begin{array}{l}H: 36-52 \\
M: 35-46\end{array}$ \\
\hline \multicolumn{4}{|l|}{ Bioquímico } \\
\hline $\begin{array}{c}\text { Colesterol Total } \\
\mathrm{mg} / \mathrm{dL}\end{array}$ & $158,2 \pm 19,1$ & $155,5 \pm 21,2$ & $<200$ \\
\hline $\begin{array}{l}\text { Triglicerídeos em Jejum } \\
\text { mg/dL }\end{array}$ & $227,5 \pm 123,5$ & $145,6 \pm 58,8$ & $<150$ \\
\hline $\begin{array}{l}\text { Lipoproteína de alta } \\
\text { densidade }(H D L) \mathrm{mg} / \mathrm{dL}\end{array}$ & $53,1 \pm 13,9$ & $47,1 \pm 10,3$ & $>40$ \\
\hline $\begin{array}{l}\text { Lipoproteína de baixa } \\
\text { densidade (LDL) mg/dL }\end{array}$ & $60,7 \pm 22,9$ & $79,1 \pm 26,7$ & $<129$ \\
\hline $\begin{array}{c}\text { T. Oxalacética (TGO) } \\
\mathrm{U} / \mathrm{L}\end{array}$ & $27,3 \pm 15,6$ & $36,8 \pm 9,9$ & Até $35 \mathrm{U} / \mathrm{L}$ \\
\hline $\begin{array}{c}\text { Transaminase } \\
\text { Glutâmica Pirúvica } \\
\text { (TGP) U/L }\end{array}$ & $23 \pm 4,1$ & $44,7 \pm 37,1$ & Até $41 \mathrm{U} / \mathrm{L}$ \\
\hline Ureia mg/DI & $29,1 \pm 16,6$ & $31,3 \pm 13,2$ & 15 a 40 \\
\hline $\begin{array}{l}\text { Creatinina } \\
\mathrm{mg} / \mathrm{dL}\end{array}$ & $0,8 \pm 0,13$ & $0,7 \pm 0,11$ & 0,4 a 1,3 \\
\hline $\begin{array}{c}\text { Proteínas Totais } \\
\text { g/dL }\end{array}$ & $7,1 \pm 0,78$ & $6,6 \pm 0,63$ & 6 a 8 \\
\hline \multicolumn{4}{|l|}{ Oxidativo } \\
\hline $\begin{array}{l}\text { Metahemoglobina } \\
(\mathrm{MeHb}) \%\end{array}$ & $2,7 \pm 0,35$ & $2,6 \pm 0,17$ & $<4,0 \%$ \\
\hline
\end{tabular}

\section{Total de participantes}

16

Legenda: *文: Média, DP: desvio padrão. Demonstração dos dados obtidos dos parâmetros hematológico, bioquímicos e oxidativo. Teste: estatística descritiva quantitativa. Fonte: Leal AR, et al., 2019.

Está representado na Figura 2, as concentrações de cianeto livre em 16 (dezesseis) amostras séricas, 8 (oito) do grupo exposto e 8 (oito) do grupo não exposto. A maior concentração média de cianeto livre foi encontrada nas amostras da população exposta, que apresentou 1,32 mg/L, com o mínimo de 1,28 e máximo de $1,36 \mathrm{mg} / \mathrm{L}$, enquanto que a população não exposta a média obtida foi de $1,29 \mathrm{mg} / \mathrm{L}$, o mínimo de 1,28 e máximo de $1,31 \mathrm{mg} / \mathrm{L}$.

Ao comparar com o valor preconizado de HCN pela via inalatória, segundo a Anseeuw K. et al., 2013, o marcador toxicológico apresentou valores moderado no sangue. E foi observada diferença estatística significativa $(p=0,0068)$, de acordo com o teste t: Amostra independente. 
Figura 2 - Representação da Concentração de HCN em grupo exposto e grupo não exposto ao cianeto no estado do Pará, 2019.

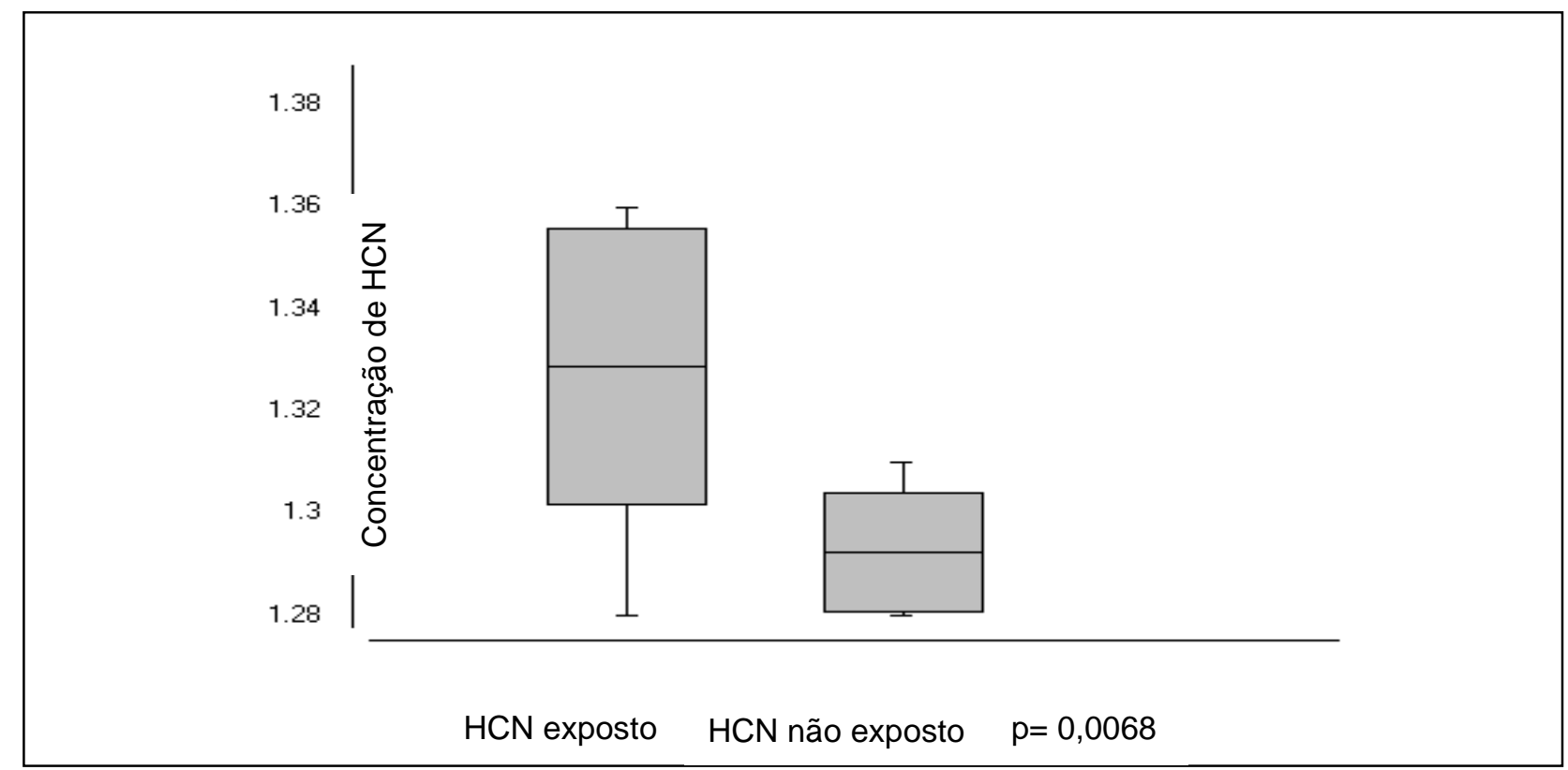

Legenda: *Teste t student HCN Livre do grupo exposto versus HCN Livre do grupo não exposto. $p=0,0068$. ${ }^{*} p \leq 0,05$. Fonte: Leal AR, et al., 2019.

A partir das análises estatísticas, foi realizado a correlação entre a concentração de HCN Livre e Metahemoglobina que pôde ser observada no Gráfico 1, comparando a exposição do grupo exposto e do grupo não exposto.

Foi observado que não houve relação significativa das duas variáveis (HCN e Mhb) do grupo exposto, já que o valor de $p$ foi de 0,8840 e o $r$ (pearson) $=0,0620$, demonstrando uma relação fraca entre ambos. $A$ mesma situação foi encontrada quando comparado a relação entre as variáveis do grupo não exposto, que apresentou o $p=0,3276$ e $r$ (Pearson) $=-0,3989$, provando uma relação fraca e indiretamente proporcional.

Gráfico 1 - Correlação linear de Pearson dos grupos estudados em Belém/Pa, 2019.
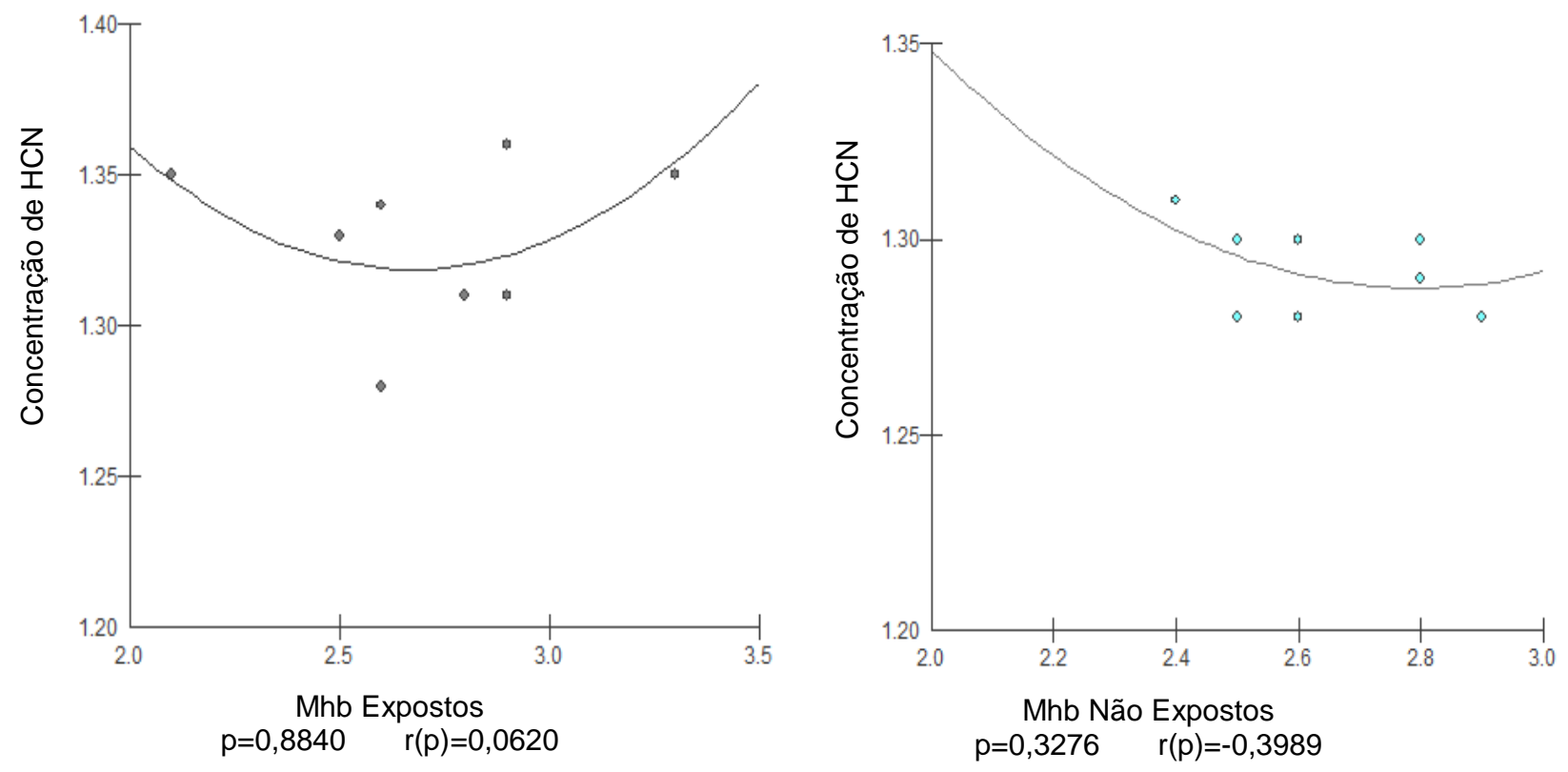

Legenda: *Teste correlação de Pearson HCN Livre e MHb do grupo exposto versus HCN Livre e MHb do grupo não exposto. $p=0,8840$ e $p=0,3276$. ${ }^{*} p \leq 0,05$. Fonte: Leal $A R$, et al., 2019. 


\section{DISCUSSÃO}

A mandioca (Manihot esculenta Crantz) apresenta destaque na dieta alimentar da população paraense, como matéria-prima para a elaboração de diversos pratos típicos, dentre eles o tacacá (CHISTÉ RC et al., 2007). A manipulação dessa iguaria torna-se um possível fator de risco aos comerciantes devido a inalação das sustâncias tóxicas contidas nos subprodutos da mandioca, como o tucupi. Já que este alimento apresenta um elevado teor de glicosídeos cianogênicos, o que confere toxicidade à raiz o que pode resultar no desenvolvimento de doenças relacionadas (CHISTÉ RC; COHEN CO, 2011).

Chisté RC et al. (2007), ao avaliar as propriedades físico-químicas de amostras de tucupi, encontraram altos teores de $\mathrm{HCN}$, o que indica a necessidade de melhorias no processo de fabricação para redução do teor desta substância, uma vez que $60 \%$ das amostras analisadas apresentaram teor total superior a $100 \mathrm{mg}$ $\mathrm{HCN}$ kg-1. Por tanto, há um risco dos comerciantes que manipulam diariamente o tucupi.

Segundo Oliveira CSB (2019) é imprescindível a identificação das variáveis sócio demográficas para identificar características associadas a determinantes que reproduzam a condição de saúde e de doença da população de estudo. As frequências entre gêneros, grau de escolaridade, renda familiar, estado civil e profissão são os que mais vem se destacando entre os fatores de riscos ao aumento da suscetibilidade a algumas patologias atualmente.

O perfil sócio demográfico dos entrevistados pôde ser observado na Tabela 1. Dos 16 (dezesseis) participantes selecionados para o estudo, $50 \%$ foram do sexo feminino e $50 \%$ do sexo masculino. A faixa etária mais frequente em ambos os grupos foi de 25 a 64 anos (62,5\%). Com relação à estado civil $75 \%$ do grupo exposto alegaram estar solteiro enquanto o grupo não exposto com $62,5 \%$. Quanto ao nível de escolaridade, $62,5 \%$ do grupo exposto relataram ter até 11 anos de estudos.

É possível que o nível de escolaridade seja um fator que está ligado diretamente à escolha da atividade laboral sem saber dos riscos inerentes a sua profissão, assim, o grupo de comerciantes de tacacá torna-se susceptível a exposição do cianeto e ao desenvolvimento de doenças relacionadas (ALMEIDA AR e LOPES JL, 2013).

Segundo Orser JR. (1999), o ofício de tacacazeira tornou-se legado e patrimônio histórico do nosso país, o que motivou o presente estudo. Cinquenta por cento (50\%) da população de estudo foram de comerciante de tacacá (expostos), enquanto a população não exposta se dividiu nas seguintes categorias: do lar, estudante, motorista, psicopedagoga, ass. Administrativo, aposentado, coletador de resíduos.

Na pesquisa foram avaliados parâmetros hematológicos e bioquímicos clínicos, com intuito de identificar alterações que pudessem ser correlacionadas com os resultados, sobretudo de marcadores de estresse oxidativo. Através da análise do hemograma dos participantes, foram observadas alterações na hemoglobina $(\mathrm{Hb})$ e hematócrito $(\mathrm{Ht})$.

Em 25\% do grupo exposto apresentou valor inferior de $\mathrm{Hb}$; e o hematócrito apresentou baixas concentrações em $25 \%$ do grupo exposto e $12,5 \%$ do grupo controle. Ao analisar as fichas dos entrevistados, verificou-se a relação da idade com as alterações dos marcadores hematológicos, predominando nos participantes com idade acima dos 40 anos, já que a anemia é a disfunção hematológica mais comumente encontrada nesta faixa etária por conta de fatores como: deficiência nutricional, doenças crônicas ou inflamação (CORONA LP et al., 2014).

Dentre o parâmetro bioquímico que se mostra alteração nos indivíduos expostos foi o triglicerídeos. Segundo o estudo de Correia JD e Perry ICS (2010) a presença de dislipidemia nas amostras séricas da população do corrente estudo pode promover a formação de colesterol e triglicerídeos ainda durante a glicólise e pode estar associada, além dos seus hábitos alimentares, a hereditariedade e 0 ambiente construído, no caso a ocupação.

Além disso, a propriedade do cianeto a ligar-se a proteínas, principalmente naquelas que estão associadas ao transporte do $\mathrm{CT}$, contribui para uma possível falha nesse transportes e/ou perda da função da proteína dentro do organismo. Fatos que precisam ser mais abordados (OLIVEIRA CSB, 2019).

Com relação aos marcadores hepáticos, renais e proteínas totais, o grupo exposto não demonstrou nenhuma alteração, resultados parcialmente compatíveis com que foi encontrado no estudo de Okafor PN et al. (2002) que demonstrou os níveis de TGP, ureia e proteínas totais dentro dos valores de referência em 
grupos exposto ao HCN. No entanto, marcadores hepáticos se mostraram alterados no grupo não-exposto, porém, pode ser justificado por fatores intrínsecos do indivíduo como estilo de vida, que inclui a obesidade, idade, consumo de álcool, estresse, sedentarismo, entre outros, os quais não foram detalhados por não serem o foco desta pesquisa. (SCHIAVO M, et al., 2003)

$\mathrm{Na}$ análise do marcador toxicológico a concentração de cianeto livre nas amostras séricas apresentou valores de $1,36 \mathrm{mg} / \mathrm{L}$ a $1,28 \mathrm{mg} / \mathrm{L}$ na população exposta e de $1,31 \mathrm{mg} / \mathrm{l}$ a $1,28 \mathrm{mg} / \mathrm{l}$ na população não exposta, evidenciando que ambos estão a um nível moderado (1-2 $\mathrm{mg} / \mathrm{L}$ ) de exposição ao cianeto (ANSEEUW $\mathrm{K}$, et al., 2013).

Os resultados encontrados nos trazem certo alerta, pois o contato dos comerciantes com os vapores produzidos no preparo do tacacá pode ser a fonte desta contaminação, e atenuada pelo consumo de outros subprodutos da mandioca, por exemplo.

Quanto a metahemoglobina, não foi observada diferença estatística com a intensidade da exposição pela via inalatória. No entanto, no grupo exposto foram encontrados os maiores valores de $\mathrm{MeHb}$, uma vez que o valor médio observado nesse grupo foi de 2,7\% MeHb, o mínimo de 2,1 \% e máximo de 3,3\% $\mathrm{MeHb}$.

De acordo com Silva JV, et al. (2016), as possíveis causas para a elevação da concentração de metahemoglobina no sangue, acima do nível padrão determinado por dosagens químicas ou enzimáticas, ocorrem pela deficiência de enzimas eritrocitárias específicas para as atividades redutoras da oxidação do ferro do grupo heme, além da indução tóxico-oxidativa da hemoglobina causada por compostos químicos oxidantes, como o cianeto.

Embora, a correlação entre essas duas variáveis, $\mathrm{HCN}$ e a MeHb, estejam diretamente ligadas como representado no gráfico 1 , os valores de $p$ e $r$ demostram que não se tem alterações estatísticas, por possuírem uma relação fraca. No entanto, observa-se que no grupo exposto há crescimento diretamente proporcional, o que se pode justificar a possível interação da volatização do cianeto encontrado no tacacá pela via inalatória.

Segundo Zacarias CH (2011), a ausência de literaturas sobre toxicidade crônica do HCN em exposição por via inalatória compromete avanços nas pesquisas, mostrando que é extremamente relevante mais estudos sobre esta temática. $O$ estudo realizado apresentou diversas limitações que dificultaram a realização do estudo.

A obtenção do número amostral desejado foi comprometida, pois, houve resistência e indisposição do público exposto a participar do estudo. Não foi possível a inclusão do teste de quantificação do tiocianato sérico, que implica na avaliação dos reais riscos de exposição ao cianeto, devido ao tempo de espera para a chegada dos reagentes. Além de que não existem dados que quantifiquem a categoria dos comerciantes de Tacacá no município de Belém/Pa, o que dificultou na busca dos participantes.

\section{CONCLUSÃO}

É inerente que os comerciantes de tacacá estão em contato com uma substância química de grande interesse toxicológico, o cianeto, pois o manuseio e a produção do tucupi apresentam teores elevados de cianeto, quando não processados corretamente. A partir dos dados obtidos percebe-se que o cianeto pode estar contribuindo com as alterações de alguns marcadores bioquimicos, em especial o triglicerídeos, que se mostrou evidente na pesquisa, além do marcador oxidativo (metahemoglobina), com níveis dentro da normalidade, que mostrou relação muito preocupante quando comparado com o HCN livre, pois quanto maior o nivel de HCN livre na circulação maior será sua interação com os eritrócitos.Por ser um estudo pioneiro da avaliação deexposiçao toxicológica, hematológicae bioquímica na população exposta ao cianeto através da sua ocupação, surge a necessidade da continuidade nos estudos, visando elucidar e compreender a contribuição dessa exposição no estado de saúde e/ou de doença da população.

\section{AGRADECIMENTO}

A Faculdade Brasil Amazônia (FIBRA) e a nossa orientadora Prof. ${ }^{a}$ Dr. a Cláudia Simone Baltazar de Oliveira por sua paciência, dedicação e conhecimento despendido conosco. Sem sua ajuda e colaboração não teríamos conseguido. 


\section{REFERÊNCIAS}

1. ALMEIDA AR e LOPES JL. Qual a relação entre escolaridade, trabalho degradante e pobreza: uma análise estatísca. VIII Encontro Produção Científica e Tecnológica. Paraná, 2013.

2. ANSEEUW K, et al. Cyanide poisoning by fire smoke inhalation: a European expert consensus. Eur J Emerg Med. 2013; 20(1): 2-9.

3. ARAÚJO JSP, LOPES, CA. Produção de farinha de mandioca na agricultura familiar, Niterói-RJ, Programa Rio Rural. Manual Técnico. 2009: 13: 17.

4. AZEVEDO FA, et al. Toxicologia e História, um triste uso da toxicidade - o zyklon. Revista Intertox-EcoAdvisorde Toxicologia Risco Ambiental e Sociedade, 2015; 8(1): 58-81.

5. BORGES MF, et al. Avaliação de Variedades de Mandioca para Consumo Humano. Pesquisa Agropecuária Brasileira. Brasília, 2002; 37 (11): 1560-1565.

6. BRASIL, Ministério da Saúde Secretaria de Atenção à Saúde. Protocolo Cínico e Diretrizes Terapêuticas. Protocolo de Uso da Hidoxocobalamina na Intoxicação Aguda por Cianeto, 2015.

7. CHISTÉ RC, et al. Estudo das Propriedades Físico-Químicas do Tucupi. Ciênc. Tecnol. Aliment. Campinas. $2007 ; 27$ (3): 437-440.

8. CHISTÉ RC, COHEN KO. Teor de cianeto total e livre nas etapas de processamento do tucupi. Rev. Inst. Adolfo Lutz, São Paulo, 2011; 70 (1): 41-46.

9. CONCEIÇÃO EC. A cultura alimentar do tacacá: um estudo em Porto Velho-ro. 2016. Monografia de Graduação. Porto Velho, RO, Brasil 2016; 56 p.

10. COOKE RD. An Enzymatic Assay for the Total Cyanide Content of Cassava (Manihot esculenta Crantz). Journal of the Science of Food and Agriculture, 1978; 29: 345-352.

11. CORREIA JD, PERRY ICS. Modulação dietética da atividade da paraoxonase: Revisão de estudos em humanos. Rev HCPA, 2010; 30(3): 271-278.

12. CORONAL LP, et al. Prevalência de anemia e fatores associados em idosos: evidências do Estudo SABE. Rev Saúde Pública 2014; 48(5):723-731.

13. DIAS VL, et al. A fabricação do tucupi e seu uso na preparação de molhos de pimenta artesanais. XVIII Encontro Nacional de Ensino de Química (XVIII ENEQ) Florianópolis, SC, Brasil - 2016.

14. ESSERS AJA, et al. Studies on the quantification of specific cyanogens in cassava products and introduction of a new chromogen. Journal of the Science of Food and Agriculture, 1993; 63: 287-296.

15. FURTADO JLB, et al. Cianeto em tiquiras: riscos e metodologia analítica. Sociedade Brasileira de Ciência e Tecnologia de Alimentos. Campinas, Brasil. 2007; 27(40): 694-700.

16. LOPES AM. Avaliação da dose letal (DL 50) oral em ratos e efeitos metabólicos da linamarina extraída de mandioca, em ratos. 2001. Dissertação (Doutorado em Agronomia) - Universidade Estadual Paulista, Botucatu, SP, $2011 ; 97$ p.

17. NAOUM PC, et al. Dosagem espectrométrica de metaemoglobina sem interferentes químicos ou enzimáticos. Rev. Bras. Hematol. Hemoter. São José do Rio Preto, 2004; 26 (1): 19-22.

18. OKAFOR PN, et al. Occupational and dietary exposures of humans to cyanide poisonig from large scale cassava processing and ingestion of cassava foods. Food and Chemical toxicology, 2002; 40; 1001-1005.

19. OLIVEIRA CSB. Respostas toxicológicas e oxidativas associadas ao consumo de peixe e farinha de mandioca em uma população rural do nordeste paraense. Tese de doutorado. Universidade Federal do Pará, Belém, $2019 ; 111$ p.

20. ORSER JR. Introdução à Arqueologia histórica. Belo Horizonte: Oficina de Livros, 1999.

21. REZENDE ALS, et al. Dieta e câncer gástrico: aspectos históricos associados ao padrão de consumo alimentar no estado do Pará. Rev. Nutr., Campinas, 2006; 19(4): 511-519.

22. SCHIAVO M, et al. Influência da dieta na concentração sérica de triglicerídeos. Jornal Brasileiro de Patologia e Medicina Laboratorial Rio de Janeiro, 2003; 39(4): 283-288.

23. SHIBAMOTO T, BJELDANES LF. Introdução à toxicologia de alimentos. 2. ed. São Paulo: Campus, $2014 ; 320$ p.

24. SILVA JV, et al. Determinação de metemoglobina em voluntários fumantes e não fumantes J Health Sci Inst. 2016;34(1):38-43.

25. SOUSA AB. Avaliação dos efeitos tóxicos do cianeto e do tiocianato no período perinatal. Estudo em ratos. Tese (doutorado) - Universidade de São Paulo. Faculdade de Medicina Veterinária e Zootecnia. Departamento de Patologia, 2004; 224p.

26. TEÓFILO TJS, et al. Toxicidade cianogênica de raízes de mandioca (manioht esculenta crantz), vendidas em dois supermercados de sobral CE. 2004. (Curso de Enfermagem) - Universidade Estadual Vale do Aracaú - UVA. Sobral, CE, 2004; $22 \mathrm{p}$.

27. ZACARIAS CH. Avaliação da exposição de trabalhadores de casas-de-farinha ao ácido cianídrico proveniente da mandioca, Manihot esculenta Crantz, no Agreste Alagoano. Dissertação (mestrado). Faculdade de Ciências Farmacêuticas da Universidade de São Paulo, São Paulo, 2011; 147 p. 